\title{
DOES POSITRONIUM FEEL DYNAMICS OF POLYMER CHAIN?
}

\author{
J. KRIŠtiaK* ${ }^{*}$ O. ŠAUŠA, P. BANDŽUCH, J. ZRUBCovÁ \\ Institute of Physics, Slovak Academy of Sciences \\ Dúbravská cesta 9, 84228 Bratislava, Slovak Republic
}

AND J. BARTO

Polymer Institute, Slovak Academy of Sciences, 84236 Bratislava, Slovak Republic

The annihilation experiments on simple elastomers at wide temperature interval of (15-370) $\mathrm{K}$ will be described. The results are consistently interpreted using free-volume models of polymer structure. The procedure of deducing some parameters of theory of polymers (e.g. viscosity, Vogel-Fulcher temperature) from the positron annihilation experiments will be shown. A great potential of positron annihilation in the study of amorphous polymers will be emphasized.

PACS numbers: $78.70 . \mathrm{Bj}, 83.80 . \mathrm{Dr}$

\section{Introduction}

According to the free volume concept of mobility in disordered media [1] a given mode may be realised in certain local regions of matrix with the lowered local density only. Therefore, the knowledge of the local structure on a scale of $10^{-7} \mathrm{~cm}$ opens up the way to better understanding of motional mechanisms, mechanical and transport properties.

We can regard glassy amorphous polymers as consisting essentially of inter-penetrating statistical coils. This coil forms occupied volume $V_{0}$. In general, unoccupied free volume $V_{\mathrm{f}}$ can be written as $V_{\mathrm{f}}=V_{\mathrm{t}}-V_{0}$, where $V_{0}$ is the volume occupied by the molecules at $0 \mathrm{~K}$ in a close-packed state and $V_{t}$ is the total volume. The fraction of free volume $f_{V}$ is then defined as $f_{V}=V_{\mathrm{f}} / V_{\mathrm{t}}$. We also assume that the free volume is not static but it is an open space that is freely moving in a medium [2].

In polymers and in other molecular materials a substantial fraction of the injected positrons forms positronium ( $\mathrm{Ps}$ ). The Ps is repelled by screened atomic nucleus and trapped in open spaces, such as holes and voids. Due to small size of

\footnotetext{
*e-mail: fyzikrjo@savba.sk
} 
Ps $(1.06 \AA)$ it is particularly sensitive to small holes in a size of $\AA$ and at a time of molecular motion from $10^{-10} \mathrm{~s}$ and longer.

In 1976 Tao [3] proposed a simple model in which the Ps particle resides in a spherical well with the radius $R$. The Ps wave function has a finite penetration depth $\Delta R$ into the material surrounding the potential well. Then $o$-Ps pick-off lifetime $\tau$ can be related to the radius of the hole $R$ as

$$
\tau=0.5\left(1-\frac{R}{R+\Delta R}+\frac{1}{2 \pi} \sin \frac{2 \pi R}{R+\Delta R}\right)^{-1} .
$$

A value $\Delta R=1.66 \AA$ is obtained by fitting (1) to the observed o-Ps lifetime of known mean hole radii in zeolites and other substances. The validity of the $\Delta R$ value is further supported by the fact that the zero-point energy of Ps atom trapped in the potential becomes equal to the binding energy of Ps $(6.8 \mathrm{eV})$ as $R$ approaches $1.66 \AA[4]$.

Equation (1) is the foundation for the determination of mean size of free-volume hole by using positron annihilation spectroscopy. This model is very rough but its application leads to reasonable conclusions, in some cases, they have been verified by another approaches.

\section{Experimental techniques and data analysis}

\subsection{Lifetime measurements}

The positron lifetime spectra are obtained by the conventional fast-fast coincidence method. Using the ${ }^{22} \mathrm{Na}$ source of $2 \mathrm{MBq}$ activity about a million counts can be measured and stored within some hours. The full width at a half maximum (FWHM) of the time resolution spectrum is $c a .300 \mathrm{ps}$. The lifetime spectra are treated by the PATFIT-88 software package of Kirkegaard et al. [5].

The relative intensity corresponding to the longest lifetime $I_{3}$ contains information related to the number of the free-volume holes. We must stress that this point is now under hot discussion. Nevertheless, a semi-empirical equation has been proposed to determine the fraction of free volume $f_{V}$ in polymers as [6] $f_{V}=C V_{\mathrm{f}} I_{3}$, where $V_{\mathrm{f}}$ is the free volume obtained from $\tau_{3}$ using Eq. (1), and $C$ is a parameter which can be determined by calibration.

The lifetime spectra measured with substantially higher statistical accuracy (several millions counts) can be analysed using the Laplace inversion program CONTIN-PALS [7]. The distribution function $\lambda \alpha(\lambda)$ is calculated from the experimental positron lifetime spectrum $N(t)$ via numerical Laplace inversion including the deconvolution of the experimental resolution $R(t)$. The $R(t)$ can be obtained from decay of ${ }^{207} \mathrm{Bi}$ nucleus. The lifetime distribution may be transformed into a hole volume density distribution $g(V)$.

\subsection{Doppler broadening measurements}

The HPGe detector with an energy resolution of $1.6 \mathrm{keV}$ for $511 \mathrm{keV}$ photon is used in Doppler broadening (DB) measurements. The $S$-parameter is used. It is defined as the sum of counts in an energy interval around the top of the photopeak divided by the total area of the peak. 
Key problem of DB measurements is a stability of electronics. Small change in energy dispersion spoils the $S$-parameter. This problem was partially solved in Ref. [8] where Gold iterative method of deconvolution compensated small shift in annihilation $\gamma$-ray spectrum.

\section{Results}

Positron lifetime and Doppler broadening measurements were carried out for set of simple elastomers having no large side groups. The basic properties of six studied samples are collected in Table I.

\section{TABLE I}

Characteristics of studied samples. PBD - poly-(butadiene), PIB - poly(isobutylene), PIP - poly(isoprene), PCIP - poly(chloroprene), a-PP - atactic poly(propylene). $\bar{M}_{\mathrm{w}}-$ molecular weight, $X_{\mathrm{c}}-$ crystallinity, $T_{\mathrm{g}}$ - glass transition temperature determined by DSC, $T_{11}$ - temperature of liquid-liquid transition.

\begin{tabular}{c|r|c|c|c}
\hline \hline Sample & \multicolumn{1}{|c}{$\bar{M}_{\mathrm{w}}$} & $T_{\mathrm{g}}[\mathrm{K}]$ & $T_{\mathrm{ll}}[\mathrm{K}]$ & $X_{\mathrm{c}}[\%]$ \\
\hline 1,4-cis-PBD & $8.1 \times 10^{5}$ & 168 & 208 & 19 \\
1,2-PBD & $2 \times 10^{5}$ & 272 & - & - \\
PIB & $4.2 \times 10^{5}$ & 203 & 263 & - \\
1,4-cis-PIP & $8 \times 10^{5}$ & 203 & 243 & - \\
1,4-trans-PClP & $7 \times 10^{5}$ & 235 & 280 & 28 \\
a-PP & $1 \times 10^{5}$ & 260 & 303 & -
\end{tabular}

Samples for measurements were in the form of discs of $10 \mathrm{~mm}$ diameter and ca. $5 \mathrm{~mm}$ thick. We used several positron sources with different activities, weaker ca. $100 \mathrm{kBq}$ and stronger $2 \mathrm{MBq}$. The activity was sealed between two $8 \mu \mathrm{m}$ Kapton foils or $3.5 \mu \mathrm{m} \mathrm{Ni}$ foils $(2 \mathrm{MBq})$. All samples were studied at wide temperature interval, from $14 \mathrm{~K}$ up to $350 \mathrm{~K}$ and more. The source-sample assembly was kept in rotary pump vacuum. The high temperature measurements (up to $470 \mathrm{~K}$ ) were performed in normal air atmosphere. During all measurements an automatic temperature regulation was used.

Several different ways of cooling and heating of samples were used. One so-called "heating" was fast cooling to $14 \mathrm{~K}$ with the cooling rate of $4 \mathrm{~K} / \mathrm{min}$. The second regime, so-called cooling, was slow cooling of samples from room temperature to $14 \mathrm{~K}$. The cooling rate is estimated to be $0.5 \mathrm{~K} / \mathrm{min}$.

Positron lifetime spectra were then measured as a function of increasing (decreasing) temperature with temperature step of 5 or $20 \mathrm{~K}$.

The Doppler broadening of annihilation line was measured simultaneously with the lifetime measurement.

A part of obtained temperature dependencies of $\tau_{3}, I_{3}$ and $S$-parameter is shown in Fig. 1 for poly(isobutylene). The sum of our results concerning $\tau_{3}$ dependence on temperature is displayed in Fig. 2. In this case we used the glass transition temperature $T_{\mathrm{g}}$ as a scaling parameter. These figures show typical 
behaviour of ortho-positronium lifetime with abrupt change of slope at the glass transition temperature $T_{\mathrm{g}}$. In all samples we observed similar behaviour. Some differences were seen at higher temperatures. We observe a constant value of $\tau_{3}$ in PClP and 1,4 PBD samples at the temperature $T \approx 1.3-1.5 T_{\mathrm{g}}$.

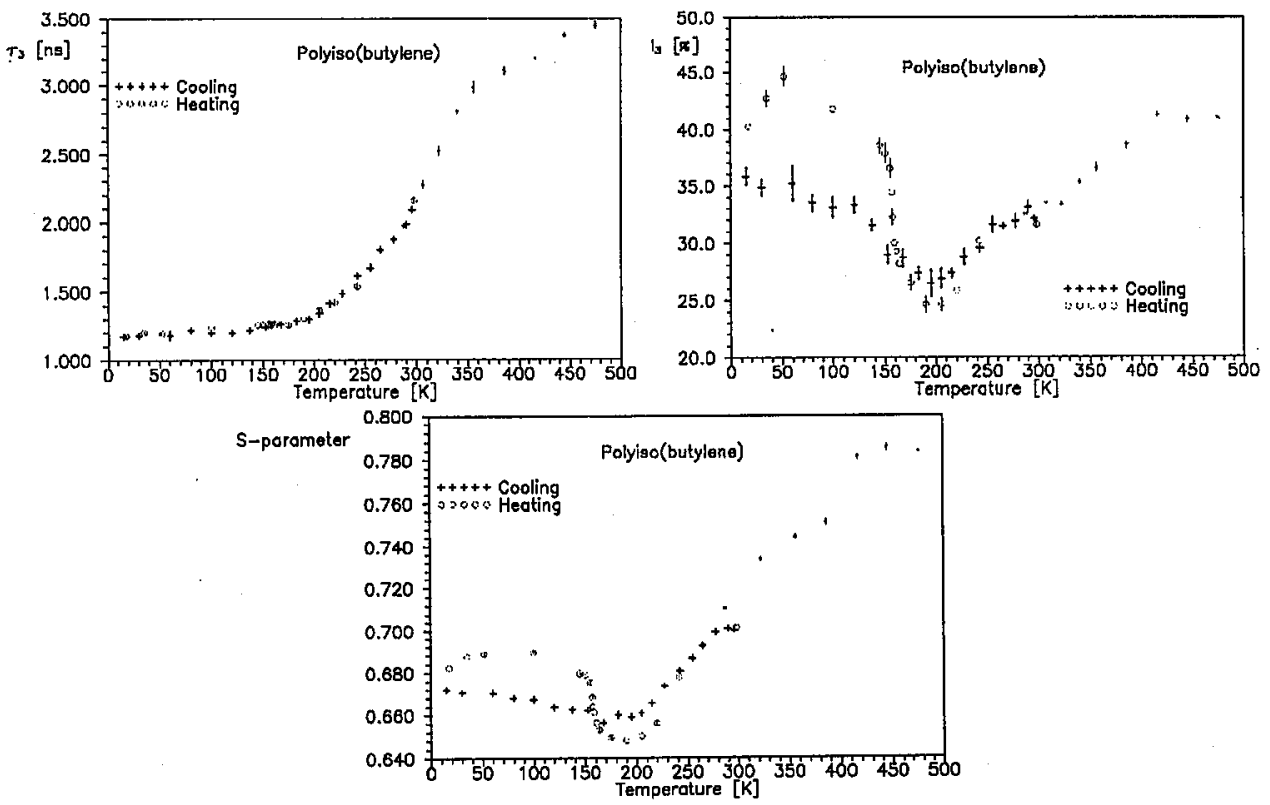

Fig. 1. The temperature dependence of positron annihilation parameters $\tau_{3}, I_{3}$, and $S$ in poly(isobutylene).

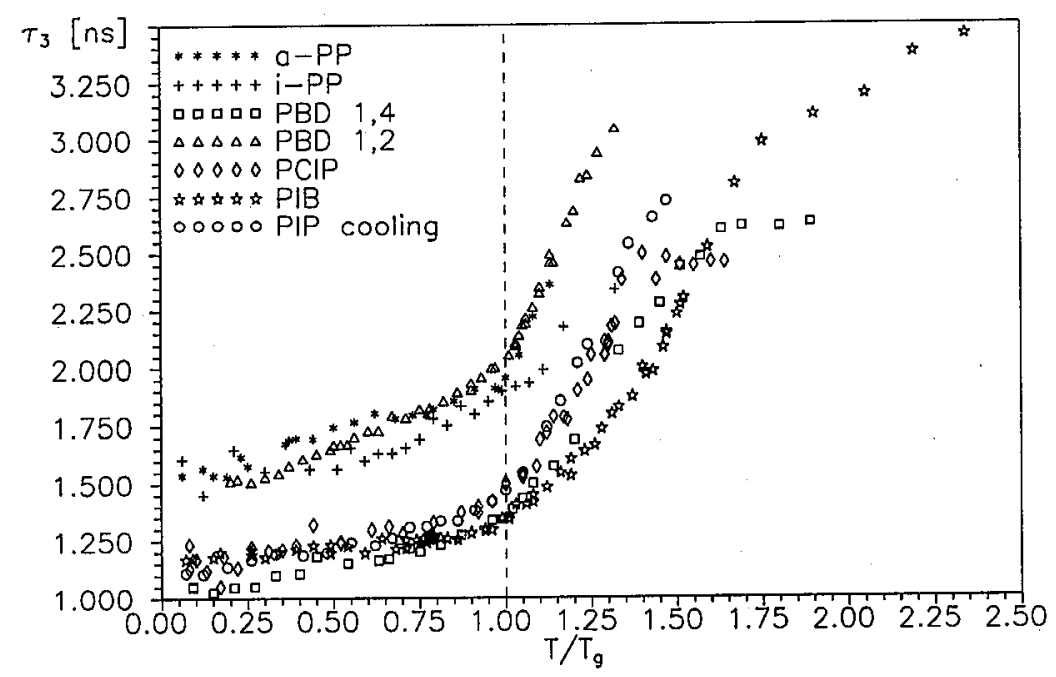

Fig. 2. The temperature dependence of $\tau_{3}, T_{\mathrm{g}}$-glass transition temperature. 

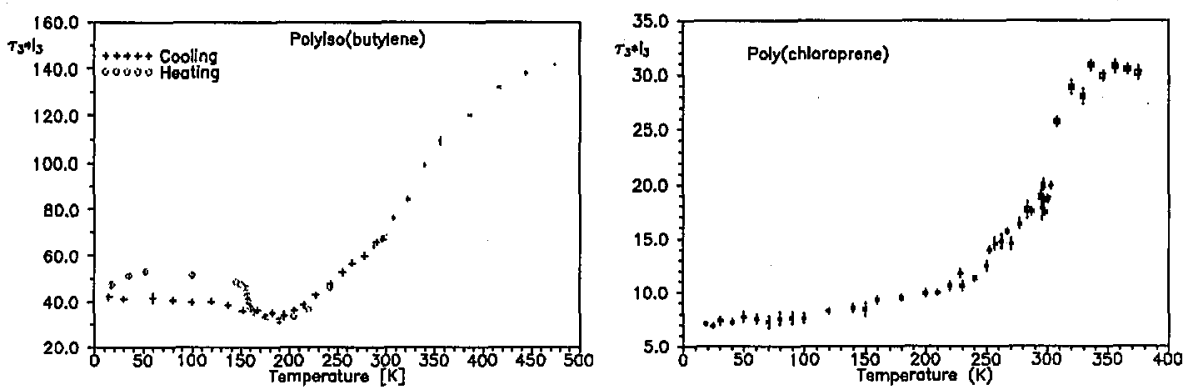

Fig. 3. The temperature behaviour of free volume fraction $f_{V}$ of poly(isobutylene) and poly(chloroprene).

The course of the intensity of the third component is more complicated especially for PIP and PIB samples.

In Fig. 3 we present temperature behaviour of the free-volume fraction $f_{V}$ of PIP and PCIP. In the case of PIB and PBD we observed that $I_{3}$ depends on a cooling rate during the measurements [9]. The $I_{3}$ is higher when the cooling rate is $4 \mathrm{~K} / \mathrm{min}$ in comparison with the rate of $0.4 \mathrm{~K} / \mathrm{min}$. Such behaviour is consistent with non-ergodic behaviour of glassy state and it is indirect argument in favour of using positronium as a detector ("seeker") of free-volume holes.

In Fig. 3 the increase in $f_{V}$ with temperature indicates the thermal expansion of free-volume hole or Ps cavity. Each curve can be approximated by neighbouring lines with a slope that can provide a value of thermal expansion coefficient of free volume $\alpha_{\mathrm{f}}$ felt by positronium atom. All slopes are normalised to the value of $f_{V}$ at $T_{\mathrm{g}}$. The mean size of the free volume holes was calculated from the mean lifetime $\tau_{3}$ with help of semiempirical relation (1). Then the thermal expansion coefficient of hole $\alpha_{\mathrm{h}}$ can also be calculated.

TABLE II

The thermal expansion coefficients of $\alpha_{\mathrm{h}}\left[\mathrm{K}^{-1}\right]$ and $\alpha_{V}\left[\mathrm{~K}^{-1}\right]$.

\begin{tabular}{c|c|c|c|c}
\hline \hline Sample & $\begin{array}{c}\alpha_{\mathrm{h}}\left(T<T_{\mathrm{g}}\right) \\
\times 10^{3}\end{array}$ & $\begin{array}{c}\alpha_{\mathrm{h}}\left(T>T_{\mathrm{g}}\right) \\
\times 10^{2}\end{array}$ & $\begin{array}{c}\alpha_{V}\left(T<T_{\mathrm{g}}\right) \\
\times 10^{4}\end{array}$ & $\begin{array}{c}\alpha_{V}\left(T>T_{\mathrm{g}}\right) \\
\times 10^{4}\end{array}$ \\
\hline 1,4-cis-PBD & 3.7 & $3.1 \pm 0.9$ & 1.9 & 7.5 \\
1,2-PBD & - & $1.4 \pm 0.2$ & - & 6.1 \\
PIB & 1.2 & $1.9 \pm 0.3$ & 1.4 & 5.7 \\
1,4-cis-PIP & 1.9 & $1.6 \pm 0.2$ & 1.9 & 5.8 \\
1,4-trans-PClP & 2.1 & $2.0 \pm 0.4$ & - & 6.1 \\
a-PP & 1.5 & $1.4 \pm 0.2$ & 1.9 & 7.1
\end{tabular}

In Table II the values of $\alpha_{\mathrm{h}}$ are summarized together with available data on the macroscopic thermal expansion coefficient $\alpha_{V}$ determined by dilatometry $[10,11]$. The data stress the need to find an explanation of systematic and almost two order difference between $\alpha_{\mathrm{h}}$ and $\alpha_{V}$.

The published simple model [12] is able to explain the values of $\alpha_{\mathrm{f}}$. 
A new model of free volume expansion was proposed [13]. The basic volumetemperature relations of model is depicted in Fig. 4. The occupied volume is expanding with the expansion coefficient $\alpha_{01}$. Thus

$$
V_{0}(T)=V_{0}\left(T_{\mathrm{g}}\right)\left[1+\alpha_{01}\left(T_{\mathrm{g}}\right)\left(T-T_{\mathrm{g}}\right)\right]
$$

and

$$
V(T)=V\left(T_{\mathrm{g}}\right)\left[1+\alpha_{V}\left(T_{\mathrm{g}}\right)\left(T-T_{\mathrm{g}}\right)\right]
$$

Algebraic manipulations give the expressions for the expansion coefficients of free volume

$$
\begin{array}{ll}
T<T_{\mathrm{g}}, \quad \alpha_{V_{\mathrm{f}_{1}}}\left(T_{\mathrm{g}}\right)=\frac{\alpha_{1}\left(T_{\mathrm{g}}\right)+\alpha_{01}\left(T_{\mathrm{g}}\right)\left[f_{V}\left(T_{\mathrm{g}}\right)-1\right]}{f_{V}\left(T_{\mathrm{g}}\right)} \\
T>T_{\mathrm{g}} \quad \alpha_{V_{t_{2}}}\left(T_{\mathrm{g}}\right)=\frac{\alpha_{2}\left(T_{\mathrm{g}}\right)+\alpha_{01}\left(T_{\mathrm{g}}\right)\left[f_{V}\left(T_{\mathrm{g}}\right)-1\right]}{f_{V}\left(T_{\mathrm{g}}\right)} .
\end{array}
$$

We can solve Eq. (2) to obtain the following relations for parameters, the expansion of occupied volume $\alpha_{01}\left(T_{\mathrm{g}}\right)$ and the free-volume fraction at $T_{\mathrm{g}}, f_{V}\left(T_{\mathrm{g}}\right)$ :

$$
\alpha_{01}\left(T_{\mathrm{g}}\right)=\frac{\alpha_{2}\left(T_{\mathrm{g}}\right) \alpha_{V_{\mathrm{t}_{1}}}\left(T_{\mathrm{g}}\right)-\alpha_{1}\left(T_{\mathrm{g}}\right) \alpha_{V_{\mathrm{t}_{2}}}\left(T_{\mathrm{g}}\right)}{\left[\alpha_{2}\left(T_{\mathrm{g}}\right)-\alpha_{1}\left(T_{\mathrm{g}}\right)\right]-\left[\alpha_{V_{\mathrm{f}_{2}}}\left(T_{\mathrm{g}}\right)-\alpha_{\mathrm{f}_{1}}\left(T_{\mathrm{g}}\right)\right]}
$$

and

$$
f_{V}\left(T_{\mathrm{g}}\right)=\frac{\alpha_{2}\left(T_{\mathrm{g}}\right)-\alpha_{1}\left(T_{\mathrm{g}}\right)}{\alpha_{V_{\mathbf{i}_{2}}}\left(T_{\mathrm{g}}\right)-\alpha_{V_{\mathrm{f}_{1}}}\left(T_{\mathrm{g}}\right)} .
$$

Very important conclusions follow from our approach:

- the combination of macroscopic dilatometric and microscopic positron annihilation data provides quantitative information on the free-volume fraction, $f_{V}$,

- there is a possibility to determine the temperature $T_{\infty}$ in which a change of free-volume entities begins, i.e. $V_{\mathrm{f}}\left(T_{\infty}\right)=0, T_{\infty}=T_{\mathrm{g}}-1 / \alpha_{V_{\mathrm{t}_{2}}}\left(T_{\mathrm{g}}\right)$.

In Table III the calculated parameters $\alpha_{01}\left(T_{\mathrm{g}}\right), f_{V}\left(T_{\mathrm{g}}\right)$ and $T_{\infty}$ are summarized. The values of Vogel-Fulcher temperature $T_{0}$ determined by relaxation,

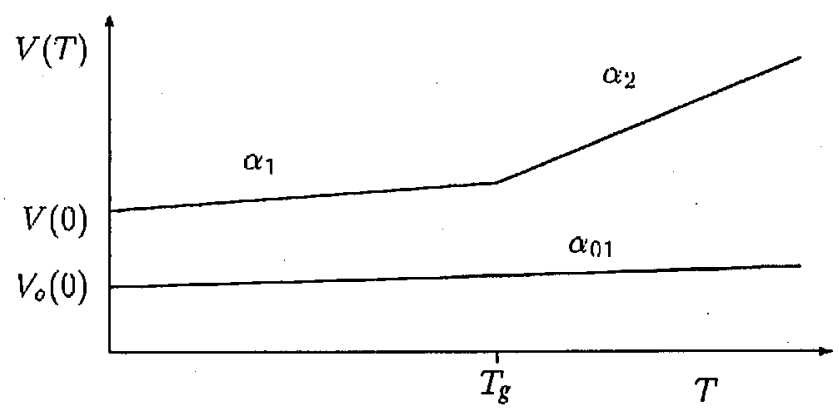

Fig. 4. The temperature behaviour of total volume $V(T), \alpha_{i}$ are thermal expansion coefficients, $\alpha_{01}$ is thermal expansion coefficient of occupied volume. 
TABLE III

The parameters of free volume calculated from model [13].

\begin{tabular}{c|c|c|c|c|c}
\hline \hline Polymer & $\begin{array}{c}\alpha_{01}\left(T_{\mathrm{g}}\right) \\
\times 10^{4}\end{array}$ & $f_{V}\left(T_{\mathrm{g}}\right)$ & $\begin{array}{c}T_{\infty} \\
{[\mathrm{K}]}\end{array}$ & $\begin{array}{c}T_{0} \\
{[\mathrm{~K}]}\end{array}$ & $\begin{array}{c}T_{\mathrm{f}} \\
{[\mathrm{K}]}\end{array}$ \\
\hline 1,4-cis-PIP & 1.39 & 0.029 & 132 & 134 & - \\
PIB & 1.24 & 0.024 & 145 & 112 & 140 \\
1,4-cis-PBD & 1.35 & 0.020 & 134 & 117 & $120 \pm 7$ \\
a-PP & 1.14 & 0.042 & 189 & 192.5 & 190 \\
1,4-trans-PClP & 1.85 & 0.021 & 180 & - & $180 \pm 10$ \\
\hline
\end{tabular}

Typical errors: $\alpha_{01}\left(T_{\mathrm{g}}\right) \pm 0.03, f_{V}\left(T_{\mathrm{g}}\right) \pm 0.001, T_{\infty} \pm 3$.

NMR or viscosity measurements [14-16] are given for comparison. The temperature $T_{\mathrm{f}}$, in which the fast process observed by quasielastic neutron scattering starts to appear, is also displayed.

Correlation among $T_{0}, T_{\mathrm{f}}$ and $T_{\infty}$ is worthy noting. Recent neutron scattering experiments [17-19] on the microscopic dynamics below and above the glass transition temperature $T_{\mathrm{g}}$ provide the Debye-Waller factor $\mathrm{e}^{2 W}, W=Q^{2}\left\langle r^{2}\right\rangle / 6$, where $Q$ is the momentum transfer of neutron and $\left\langle r^{2}\right\rangle$ is the mean squared displacement of scatterer. The temperature behaviour of $\left\langle r^{2}\right\rangle$ is linear below as well as above $T_{\mathrm{g}}$. The relative slopes of $\left\langle r^{2}\right\rangle$ (the expansion coefficient of $\left\langle r^{2}\right\rangle$ ) have been calculated. They are summarized in Table IV.

The comparison of Table II and IV shows remarkable resemblance of the rate of change of two geometrical quantities $\left(\alpha_{\mathrm{h}}\right.$ and $\left.\alpha\left(\left\langle r^{2}\right\rangle\right)\right)$ with temperature.

\section{TABLE IV}

The rate of change of the mean squared displacement $\left\langle r^{2}\right\rangle$ with temperature $T$.

\begin{tabular}{c|c|c}
\hline \multirow{2}{*}{} & \multicolumn{2}{|c}{$\alpha\left[\left\langle r^{2}\right\rangle\right]\left[\mathrm{K}^{-1}\right]$} \\
\cline { 2 - 3 } & $T<T_{\mathrm{g}}$ & $T>T_{\mathrm{g}}$ \\
\hline 1,4-PBD & $5.9 \times 10^{-3}$ & $3.7 \times 10^{-2}$ \\
PIB & $4.9 \times 10^{-3}$ & $5.5 \times 10^{-2}$ \\
PClP & $4.5 \times 10^{-3}$ & $1.8 \times 10^{-2}$ \\
a-PP & $3.9 \times 10^{-3}$ & $3.1 \times 10^{-2}$
\end{tabular}

It was said that dynamical properties and consequently, various physical and physico-chemical ones are determined not only by the mean free-volume hole size and its concentration but also by the free-volume hole size distribution. Figure 5 shows an example of dependence of the free-volume hole distribution on temperature for a typical amorphous polymer - PIB as obtained using CON- 


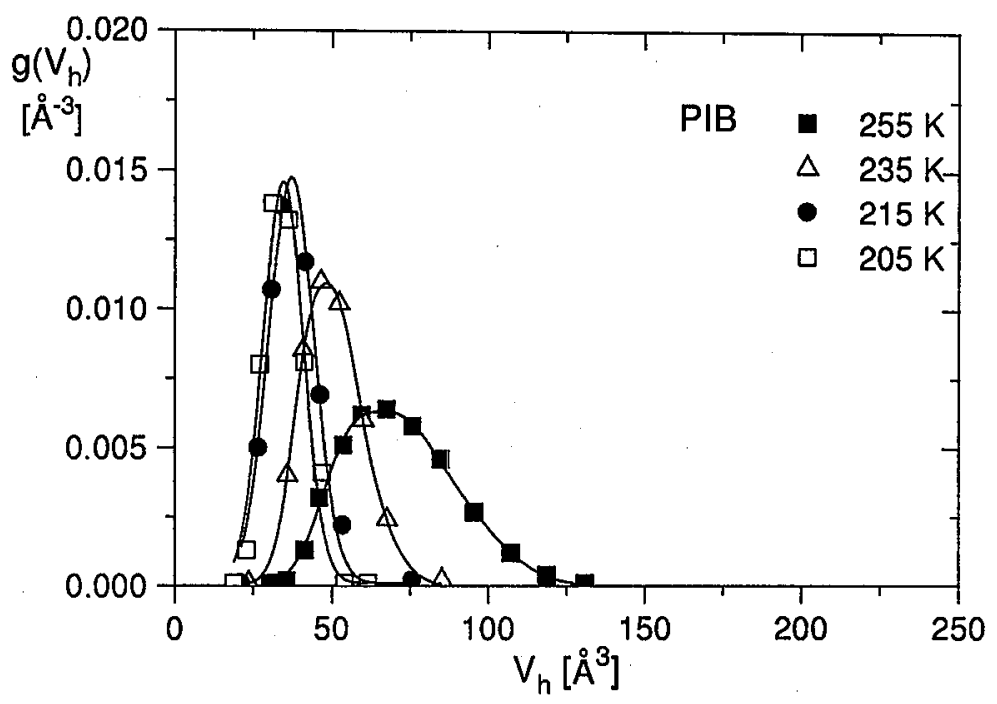

Fig. 5. Free volume distribution $g\left(V_{h}\right)$ of poly(isobutylene) at the temperature 205, $215,235,255 \mathrm{~K}$.

TIN programme. Qualitatively, the maxima of distributions shift to higher values with increasing temperature. Simultaneously, widening of distribution occurs, which is small in lower temperature range and pronounced at higher temperatures, especially well above $T_{\mathrm{g}}$. Analogical trends were observed in other elastomer cis-1,4-poly(butadiene) [20].

\section{Conclusion}

We have discussed our most recent results on probing the free volume in polymers by means of positron annihilation lifetime (PAL) spectroscopy. We found many encouraging correlations between results of positron annihilation, quasielastic neutron scattering and more traditional methods (viscosity, NMR and so on). We have formulated the hypothesis explaining up to two order difference in the expansion of the bulk substance and the microscopic free volume.

This research is supported by grants from Grant Agency of Slovak Academy of Sciences $(2 / 5082 / 98$ and 2/4008/97). One of us (J.K.) wishes to thank all his students and collaborators.

\section{References}

[1] R. Zallen, Physics of Amorphous Solids, Wiley, New York 1986.

[2] M.H. Cohen, G.S. Grest, Phys. Rev. B 20, 1077 (1979).

[3] S.J. Tao, Appl. Phys. 10, 67 (1979).

[4] K. Hirata, Y. Kobayashi, Y. Ujihira, J. Chem. Soc., Faraday Trans. 92, 985 (1996).

[5] P. Kirkegaard, M. Eldrup, E. Morgensen, N.Y. Pedersen, Comput. Phys. Comm. 23, 307 (1989).

[6] Y.Y. Wang, H. Nakanishi, Y.C. Jean, T.C. Sandreczki, J. Polym. Sci. B 28, 1431 (1990). 
[7] R.B. Gregory, Y. Zhu, Nucl. Instrum. Methods Phys. Res. A 290, 172 (1990).

[8] P. Bandžuch, M. Morháč, J. Krištiak, Nucl. Instrum. Methods Phys. Res. A 384, 506 (1997).

[9] J. Krištiak, O. Šauša, P. Bandžuch, J. Bartoš, J. Radioanal. Nucl. Chem. A 210, 563 (1996).

[10] L.A. Wood, J. Polym. Sci. 28, 319 (1958).

[11] D.L. Beck, A.A. Hiltz, J.F. Knox, Trans. Soc. Plastic Eng. 3, 279 (1963).

[12] J. Krištiak, J. Bartoš, O. Šauša, P. Bandžuch, Mater. Sci. Forum 255-257, 35 (1997).

[13] P. Bandžuch, Ph.D. Thesis, Comenius University, Bratislava 1997.

[14] A. Dekmezian, D.E. Axelson, J.J. Dechter, J. Polym. Phys. 23, 367 (1985).

[15] G.C. Berry, T.G. Fox, Adv. Polym. Sci. 5, 261 (1968).

[16] C.M. Roland, P.G. Santageev, Z. Baran, J. Runt, Macromolecules 27, 5380 (1994).

[17] T. Kanaya, T. Kawaguchi, K. Kaji, J. Chem. Phys. 105, 4342 (1996).

[18] T. Kanaya, T. Kawaguchi, K. Kaji, Physica B 182, 403 (1992).

[19] T. Kanaya, K. Kaji, J. Bartoš, M. Klimová, Macromolecules 30, 1107 (1997).

[20] J. Bartoš, P. Bandžuch, O. Šauša, K. Krištiaková, J. Krištiak, T. Kanaya, W. Jenninger, Macromolecules 30, 6906 (1997). 